\title{
Quand la locution en un mot marque une glose de nomination : quelques effets de matérialité
}

Corinne Gomila ${ }^{1}$

Université de Montpellier, Praxiling UMR 5267-CNRS

\begin{abstract}
Résumé. Cet article porte sur le déploiement en discours de l'expression en un mot. Notre travail aborde en premier lieu l'expression en elle-même afin de mieux comprendre en quoi elle est, plus que toutes autres, propre à signifier la matérialité verbale ; puis l'étude examine le fonctionnement de la locution en discours au sein d'une configuration privilégiée dans le corpus, la glose de nomination, avant d'en sonder la dimension sylleptique et le double-jeu.
\end{abstract}

\begin{abstract}
Naming gloss stated with in a word: using signs and meaning. This article deals with the locution in a word used in discourse. At the first step, we want to understand how this adverb phrase performs with linguistic materiality; then the study examines the type of gloss written in that form that we can call $a$ naming gloss. Finally, the last step is aimed at establishing the typical features of that naming gloss: syllepse and autonymous character.
\end{abstract}

\section{Introduction}

Au fil de son discours, un locuteur peut être amené à parler de sa propre énonciation. Il peut la renforcer, la spécifier, la commenter, en un mot, exprimer différentes attitudes vis-à-vis de ce qu'il est en train de dire. Ce marquage énonciatif est une valeur ajoutée qui conditionne le sens de l'énoncé et son entendement.

Nombre de « petits mots », d'expressions plus ou moins figées assurent cette fonction au premier rang desquels figurent notamment les expressions énonciatives (Perrin, 2012) et les marqueurs discursifs (Dostie et Lefeuvre, 2017). Si plusieurs travaux se sont récemment centrés sur les marqueurs formés sur le verbe dire (Rouanne et Anscombre, 2016 ; GómezJordana Ferary et Anscombre, 2015), peu d'études à notre connaissance ont pris pour objet principal les expressions formées sur le nom mot.

Ancrée dans le cadre théorique de l'énonciation en général et en particulier dans le champ du métalangage naturel, s'appuyant sur les travaux de J. Authier-Revuz ${ }^{1}$, (1994, $1995)$ fondamentaux sur le sujet, et inspirée par ceux d'A. Steuckardt $(2003,2005,2006)$ et 
de C. Julia (2001) portant spécifiquement sur la glose, cette étude veut questionner le jeu de la matérialité et du sens que peut susciter en discours l'expression en un mot.

Le corpus utilisé provient de la base Frantext qui répertorie 7122 occurrences. Seules sont retenues pour l'analyse les formes cristallisées qui disent plus ou autre chose que la seule combinaison de leurs constituants. Sont écartées par conséquent les occurrences qui comme dans l'exemple 3 ont un sens purement descriptif : les éléments en, un et mot s'entendent séparément et sont régis par le verbe transformer; sont conservées en revanche toutes celles qui composent une expression autonome et supprimable assurant la prise en charge énonciative d'un énoncé qu'elles modalisent. Cet énoncé est alors représenté comme dit en un mot, ce qui est le cas de l'adjectif légère en 1, et de l'assertion il a l'autorité en 2.

(1) Qu'ils sont nobles, alors, vénérables, ces grands possesseurs de trésors qui furent jadis, dans leurs vingt ans, comme tout le monde des gambadeurs, des âmes jeunes, primesautières, insupportables, en un mot légères. (Barrès, Mes cahiers, 1911)

(2) Il a contre lui les faux érudits et les érudits trop entêtés d'érudition. Il n'a pour lui ni les frivoles ni les sensibles ou les nerveux. Les femmes le lisent peu. Les sympathies qu'il inspire sont rares et austères. Avec cela, il est quelqu'un ; son avis compte, on sent qu'il n'est jamais négligeable. En un mot, il a l'autorité. (Lemaître, Les Contemporains, 1885)

(3) $[\ldots]$ Changui, en dialecte haut-rhinois, est un diminutif de Jean. Quand je dis un diminutif... C'est juste le contraire, au lieu d'alléger, il écrase, il transforme une syllabe mélodieuse en un mot glapissant. (Egen, Les Tilleuls de Lautenbach, 1979)

Il s'agira tout d'abord d'analyser l'expression en un mot pour mieux comprendre en quoi elle est, plus que toutes autres, apte à signifier le monde des signes ; puis de l'examiner en discours au sein d'une configuration privilégiée dans le corpus - la glose - avant de sonder pour cette figure, et pour ce marqueur, les effets en discours de leur matérialité.

\section{Caractérisation de la locution : une prédisposition à l'expression de la matérialité}

En dehors de tout contexte, l'expression en un mot signifie déjà, et en elle-même, la «consistance» linguistique du discours; elle exprime via la présence du terme mot l'existence des unités de la langue.

\subsection{Le poids du mot}

Sur le plan grammatical, l'expression se présente comme un groupe prépositionnel qui enchaine à la préposition en à valeur englobante, un déterminant un et un nom mot. Elle présente une structure figée qui admet toutefois des variantes paradigmatiques : en deux mots, en trois mots.

L'ensemble constitue une unité lexicale, une locution adverbiale apte à fonctionner comme un adverbe de constituant, mais surtout comme un adverbe d'énonciation (Anscombre, Donaire, Haillet, 2013) :

En un mot peut figurer en position intrapropositionnelle et tenir le rôle d'un modifieur postposé au groupe verbal : 
(4) Je lui en dis mon avis en un mot, et continuai. Il me dit pour raison qu'il n'était non plus en état d'écrire que de parler. Je ne voulus pas contester. (Saint-Simon, Mémoires, 1829)

Dans ce cas, la locution adverbiale caractérise un élément de la phrase (dire son avis). Elle se prête à l'extraction en c'est...que : "c'est en un mot que je lui en dis mon avis »; elle reste dans la portée de la négation : « je ne lui en dis pas mon avis en un mot (je lui en dis mon avis, mais pas en un mot) » et elle commute avec un adverbe de constituant comme rapidement.

En position extrapropositionnelle, à l'initiale ou en médiane, en un mot se comporte comme un adverbe d'énonciation qui ne modifie pas un élément de l'énoncé, mais affecte la phrase dans son ensemble en la commentant.

(5) Quant aux critiques, je ne vous en fais aucune, parce que je ne suis pas sûr de les penser moi-même, dans un quart d'heure. J'ai, en un mot, peur de dire des inepties, dont j'aurais un remords immédiat. (Flaubert, Correspondance, 1857)

Dans ce cas, le syntagme adverbial est employé pour caractériser non pas le contenu de la phrase ou l'un de ses constituants, mais l'acte même de dire et la manière de le faire. Elle ne peut être extraite par c'est...que : "*c'est en un mot que j'ai peur de dire des inepties »; elle reste hors de portée de la négation : «je n'ai pas, en un mot, peur de dire des inepties ». Elle commute avec la locution en résumé qui, comme elle, caractérise la dimension synthétique du dire.

Notons également, dans la composition de la séquence en un mot, le caractère ambivalent du déterminant. À la différence de l'anglais qui dispose de deux termes pour distinguer le numéral de l'indéfini - in a word vs in one word - seul le contexte permet en français d'interpréter un comme un indéfini ou un numéral et d'actualiser ainsi le sens à donner au substantif mot.

En effet, mot n'est pas un terme ordinaire sur le plan sémantique comme au niveau sémiotique :

Polysémique, il peut signifier tout autant une « unité porteuse de signification » qu'un « ensemble de mots constituant un énoncé » ${ }^{2}$ (TLFi). De fait, quelle que soit l'ampleur de l'énoncé en jeu - légère en 1 ou J'ai peur de dire des inepties, dont j'aurais un remord immédiat en 5 -, la locution reste opératoire et son emploi s'avère pertinent dans les deux cas.

Cette polysémie génère, comme nous le verrons, du jeu entre matérialité et sens car si le fil du discours centre naturellement l'attention du lecteur sur le contenu du propos, à tout moment et au moindre indice, le « sentiment linguistique profane » (Lecolle, 2014) de cet usager de la langue peut être activé et joué en faveur de l'acception métalinguistique du terme.

De fait, la présence du nom mot donne à la locution adverbiale une valeur intrinsèquement métalinguistique, ce qui la distingue de ses synonymes, bref ou en somme. Sur le plan sémiotique mot est un terme " destiné à parler du langage » pour reprendre la formulation de J. Rey-Debove (1997, 26-36). Il fait partie des « noms métalinguistiques d'unité » comme phrase, syllabe ou verbe, et en tant que tel, il constitue " un signe de multisigne " puisqu'il signifie un signe dont le signifiant et le signifié sont des «variables solidaires ». Ainsi, mot peut fonctionner comme un présentateur d'autonyme, un catégorisateur métalinguistique : dans l'exemple 2, légère pourrait sans dommage être remplacé par d'autres unités qui souscrivent aux propriétés définitoires $d u$ mot, mais non par la lettre $m$ ou le chiffre 5 . 
L'ensemble de ces caractéristiques linguistique et métalinguistique prédispose la locution en un mot à des emplois en discours principalement méta-énonciatifs.

\subsection{Dans le champ énonciatif, un « rappel de langage »}

La locution participe d'un « rappel du langage » dans l'énonciation (Authier-Revuz, 1995, II). Elle apparait dans le corpus comme une expression méta-énonciative polyvalente. Selon l'intention de l'énonciateur, et porté par des structures différentes, en un mot peut tenir le rôle d'un modalisateur, d'un marqueur discursif ou d'une glose (Gomila, 2019) à l'instar des trois extraits suivants :

(6) Françoise Baudoin était, en un mot, un de ces êtres purs, ignorants et crédules, qui peuvent, quelquefois à leur insu, devenir des instruments terribles entre d'habiles et dangereuses mains. SUE Eugène - Le Juif errant (1845)

(7) Une femme qui n'est pas capable de réflexions, trouve quelque soulagement dans la petitesse même de ses vues. Elle ne sort point du tourbillon qui l'environne. Elle ne voit rien au-delà du présent. En un mot, elle ne pense point. Prévost L'Abbé, Lettres angloises ou Histoire de miss Clarisse Harlove, 1751)

(8) Elles sentent leur faiblesse ; de là, le besoin de plaire [...]; de là, cette continuelle observation de tout ce qui se passe autour d'elles ; de là, leur dissimulation, leurs petits manèges, leurs manières, leurs grâces, en un mot leur coquetterie, qui, dans l'état social actuel, doit être regardée comme la réunion, ou le résultat de leurs bonnes et de leurs mauvaises qualités. Cabanis, Rapports $d u$ physique et du moral de l'homme, 1808)

Ainsi en un mot dans l'exemple 6 fonctionne comme un modalisateur simple signifiant en surplomb le caractère condensé du dire en train de s'énoncer et supposant dialogiquement d'autres façons plus détaillées d'exprimer la même chose.

Dans l'exemple 7, la locution souscrit entièrement aux critères du marqueur discursif définis par L. Rouanne et J.-C. Anscombre (2016, 5-6). Elle constitue une « entité non notionnelle dans son fonctionnement » qui inclut l'attitude du locuteur sur « le mode de la monstration », bénéficie d'une certaine « autonomie syntaxique, sémantique et prosodique ", se caractérise " par la perte du caractère référentiel de certains de ces composants », et s'avère " très généralement non compositionnel ». En tant que marqueur discursif, la locution en un mot figure en position détachée, ici en position initiale. Remplissant alors tous les critères d'un adverbe d'énonciation, elle fonctionne dans cet exemple non pas comme un marqueur de reformulation ordinaire, mais comme un « connecteur de reformulation non paraphrastique » (Roulet, 1987 ; Rossari, 1990). En effet, en un mot n'instaure pas de relation d'équivalence stricte dans le couplage effectué, mais opère un changement de perspective énonciative : la locution reprend d'une part une suite de propositions - «Elle ne sort point du tourbillon qui l'environne. Elle ne voit rien au-delà du présent »; elle introduit d'autre part sur le même moule syntaxique, une reformulation substituable, mais non équivalente : la reformulation « elle ne pense point »a une valeur récapitulative et conclusive que n'ont pas les formulations précédentes.

Non sans similitudes, mais opérant différemment en 8 , la locution, intégrée syntaxiquement, précède ou suit directement le mot, le dernier d'une série sur lequel elle vient « se boucler ». Ce faisant, elle objective ce mot sans jamais rompre le fil du discours en cours. Le terme modalisé - unité ou syntagme - est le dernier d'une suite figurant une recherche de mot. 
C'est au sein de cette configuration singulière, où la locution ajoute un mot à une suite de termes, dans cette tournure particulièrement bien représentée dans le corpus aux périodes classique et moderne, que la séquence en un mot sera désormais observée.

\section{Une configuration privilégiée : la glose}

Ce type de configuration relève de la classe des gloses. Une glose se compose habituellement de trois éléments : entre le segment glosé et la glose elle-même s'intercale un élément de liaison, un marqueur de glose, ici la locution en un mot.

Au sens large, elle constitue une opération méta-énonciative faite par l'énonciateur, portant sur un élément de son discours et visant à en expliciter le sens ou la référence. Cet élément peut être un syntagme, une phrase ou un texte, mais, dans une acception plus étroite comme celle retenue par C. Julia ou A. Steuckardt, la glose revient sur une unité lexicale.

L'étude de C. Julia (2001) se centre sur un type de glose particulier dont elle donne une représentation systémique, la glose de spécification du sens. Celle-ci procède d'une opération énonciative de sélection et de stipulation du sens ou des sens d'un mot en discours. Privilégiant « le rapport du sujet à la langue et à l'infini des discours, engendrant sans cesse des valeurs nouvelles qu'ils reversent à la langue » $(2001,14)$ l'auteure caractérise huit modèles de gloses de spécification du sens comme le modèle diachronique (au sens étymologique du terme, au sens latin...) ou orthonymique (au sens propre du terme, au sens littéral ...) pour ne citer que les deux premiers.

Les travaux successifs d'A. Steuckardt $(2003,2005,2006)$ traitent de la glose de mot. En étudiant les gloses du locuteur, l'auteure cherche avant tout à " recueillir des informations sur le lexique » $(2006,7)$ qui lui permettront notamment de dresser une typologie sémiotique, syntaxique et pragmatique des marqueurs de glose. Sont ainsi distingués parmi les éléments de liaison structurant la glose, des marqueurs d'indication de « dénomination » (appelé), des marqueurs de "signifié » (ce qui veut dire), d' " exemplaires remarquables » (par exemple, en particulier...) ou de «nomination nouvelle » (à savoir, autrement dit, ou plutôt ...).

Ces deux études sont précieuses qui aident à la caractérisation des gloses formées sur en un mot.

\subsection{Une glose de nomination complexe}

De fait, cette forme de glose ne constitue pas une glose de signification : ainsi, bien qu'elle induise un effet explicatif, la glose de l'exemple 9 " vide de sens, faible, en un mot féminin " n'a pas pour vocation première d'indiquer le signifié d'un terme ou d'en fixer le sens spécifique.

(9) Absorbé par ces idées sévères, le peu qu'il daignait comprendre des mots obligeants des deux amies lui déplaisait comme vide de sens, niais, faible, en un mot féminin. (Stendhal, Le Rouge et le noir, 1830)

La relation de couplage assurée par l'élément de liaison dans la linéarité du discours n'est pas une relation définitoire. D'ailleurs, lorsque l'énonciateur veut signifier cette spécification du sens, il ajoute comme en 10 une glose supplémentaire, dans le pire sens $d u$ mot.

(10) Pour ces deux causes, à cette époque de la vie si gaie pour les autres enfants, j'étais méchant, sombre, déraisonnable, esclave en un mot, dans le pire sens du mot, et peu à peu je pris les sentiments de cet état. (Stendhal, Vie de Henri Brulard, 1836) 
Ce type de glose procède en fait d'une opération de nomination qui, en figurant une recherche de mots, veut nommer au plus près la chose visée. En un mot s'inscrit de ce fait dans la catégorie « des marqueurs d'indication de nouvelle nomination » isolée par

A. Steuckardt (2006). Cependant, le processus de nomination à l'œuvre dans cette configuration résomptive s'avère bien plus complexe qu'il n'y parait :

D'une part, parce que la nomination est multiple. En 9 comme en 10, la séquence manifeste "une équivalence (syntaxique et référentielle) entre plusieurs manières de nommer une même chose donnant lieu à des nominations partagées » (Bikialo, 2006 :55). Notons toutefois que la locution en un mot met en perspective, non pas deux nominations sémantiquement équivalentes, comme peuvent le faire par exemple les marqueurs en d'autres termes ou autrement dit, mais une nomination autre, voire meilleure que les précédentes. Aussi, de part et d'autre du marqueur, ces nominations n'ont pas strictement la même valeur. Dans l'extrait 10, le terme féminin a d'une certaine façon plus de pertinence référentielle et plus de poids sémantique que les trois nominations qui la précèdent et qu'il recouvre.

D'autre part, parce qu'au niveau sémiotique et énonciatif, le marqueur en un mot fait du signe qu'il glose une occurrence singulière, chargée d'un cumul sémiotique : féminin en 9 et esclave en 10 sont à la fois employés et mentionnés dans ces extraits. D'un point de vue sémiotique, si le signe féminin réfère normalement tout autant que les termes vide de sens, niais et faible, il est aussi un signe autonymisé par le commentaire métalinguistique du marqueur. Au plan énonciatif, cette classe de gloses de nomination manifeste une modalisation particulière, une « modalisation autonymique » du dire définie par

J. Authier-Revuz (1995:33) comme « un mode dédoublé opacifiant du dire, où le dire (1) s'effectue, en parlant des choses avec des mots, (2) se représente en train de se faire, (3) se présente, via l'autonymie, dans sa forme même ».

Cette forme de glose construite avec le marqueur en un mot constitue une glose de nomination complexe dont la particularité est également de progresser à vue.

\subsection{Une glose de nomination « à progression montrée »}

J. Authier-Revuz $(1995,618-619)$ a été la première à notre connaissance à décrire ce processus de nomination qui s'affiche "à ciel ouvert». Enchainant une série de termes, le mouvement de nomination s'effectue en progressant vers le mot ultime donné comme le meilleur. Les termes essayés dans l'extrait suivant ne sont pas forcément synonymes : inquiète, tenace et orgueilleuse ne partagent aucun sème commun. Les adjectifs inquiète et ombrageuse ont des sens voisins, mais ne correspondent pas mieux au sens de l'adjectif orgueilleuse.

(11) Cela est bien visible dans les lettres d'un voyageur. On y sent la personnalité ardente de la jeunesse, inquiète, tenace, ombrageuse, orgueilleuse en un mot. Oui, orgueilleuse, je l'étais, et je le fus encore longtemps après. (Sand, Histoire de ma vie, 1855)

Dans ce processus de nomination, il s'agit de nommer au plus juste. Le terme que la locution modalise se présente alors comme plus proche, plus précis ou plus couvrant, bref, bien plus apte que tous les précédents à désigner la chose visée.

Cependant, ce type de nomination « à progression montrée »(Authier-Revuz, 1995, 619) peut aussi témoigner de l'écart qui sépare le mot de la chose ciblée. On relève ainsi dans le corpus différents degrés d'ajustement : le dernier mot peut être présenté comme serrant au plus près à la chose que l'on veut désigner. Il peut faire mouche et être validé 
comme en 11 par l'énonciateur « Oui, orgueilleuse, je l'étais ». Mais il peut aussi rendre compte d'une approximation signifiée par l'alternative (13), la totalité globalisante du pronom tout (14), voire même signaler un manque de mot, une nomination par défaut (15), ce que montrent respectivement les trois gloses suivantes :

(12) Tout est hors de prix maintenant ici. Tout est vol, mensonge, luxe couvrant l'indigence, privation ou ruine, en un mot. (Sand, Correspondance, 1842)

(13) Je l'ai retirée de l'état le plus déplorable qui fût jamais : je lui ai sacrifié épouse, honneur, fortune : tout, en un mot. (Godard D'Aucour, Mémoires turcs, 1743)

(14) Sa fantaisie devenait une idée fixe, une perpétuelle rêverie, une souffrance fiévreuse, une passion en un mot, puisque nous l'avons nommée ainsi, faute d'un nom qui peignît cette affection à la fois brutale et romanesque, particulière à la situation et à la nature d'esprit de notre jeune personnage. (Sand, Jeanne, 1844)

Au terme de cette section, il apparait que la locution en un mot fonctionne effectivement comme un marqueur de gloses. Les gloses structurées par ce marqueur sont des gloses de nomination complexes caractérisées par plusieurs traits distinctifs : une structure de couplage syntaxique à trois éléments coordonnés - mots glosés, glose et marqueur ; un processus de nomination résomptive typique s'effectuant à «multi-termes » et à « progression montrée » (Authier-Revuz, 1995, 618-619) ; un mouvement de modalisation autonymique affirmé, opéré par le marqueur qui fait de la glose proprement dite un signe « opacifié », à la fois employé et mentionné.

Dans cet emploi, la locution fonctionne comme un marqueur de glose de mots. Il s'ajoute à la liste des seize marqueurs étudiés par A. Steuckardt (2006) et s'intègre naturellement dans la classification proposée : marqueur de glose à métaterme opérant une nouvelle nomination résomptive.

\section{Jouer de la matérialité en discours}

Cette dernière section examine les effets de la matérialité propre à ce format de glose : sa dimension figurale d'une part et la spécificité du marqueur d'autre part.

\subsection{Une glose à dimension figurale}

Ce qui frappe dès lors que l'on examine les 7122 occurrences de la locution en un mot, c'est la saillance de cette configuration. Bien qu'elle ne soit pas la plus fréquente ${ }^{3}$ au sein du corpus, elle est repérable au premier coup d'œil dans le défilement du concordancier de Frantext. La glose de nomination résomptive se distingue par sa dimension figurale : elle se présente en effet comme une forme discursive « marquée, libre et mesurable qui renforce le rendement des énoncés ». (Bonhomme, 1998, 6).

La configuration de la glose de nomination apparait tout d'abord fermement structurée. Elle connait deux variantes, deux moules syntaxiques, que l'on pourrait symboliser par les formules $x^{n}$ en un mot z' et $x^{n} z^{\prime}$ en un mot où l'exposant $n$ désigne une série d'unités et l'apostrophe le cumul sémiotique de l'autonyme.

(15) [...] c'est essentiellement à ses propres efforts qu'elle doit les résultats qu'elle enregistre aujourd'hui : discipline économique, rigueur financière, probité fiscale, en un mot austérité. (Mendès-France, Euvres complètes, 1985) 
(16) [...] car vous ne pouvez réitérer indéfiniment les heures canoniales et il faut travailler, manger, vivre, en un mot. (Huysmans, L'Oblat, 1903)

Sur la chaine du discours, la glose se perçoit d'emblée en raison de l'agencement inhabituel des mots : la répétition d'un même type de syntagme à la même place syntaxique - duplication de groupes nominaux de même facture en 15 ; redondance d'infinitifs en 16 marque singulièrement l'énoncé d'un effet d'amplification. Transgressant les lois ordinaires du langage, et la maxime de quantité en particulier (Grice, 1979), l'énoncé joue de l'accumulation et de la gradation de ses unités ${ }^{4}$ de part et d'autre du marqueur :

La suite de mots en amont peut être restreinte, limitée à deux éléments, mais elle cumule en général entre trois et quatre nominations distinctes. Les unités peuvent être de même nature, sans que cela soit systématique ${ }^{5}$. Le plus souvent, ce sont des noms ou des adjectifs, parfois des verbes comme en (16). Il peut s'agir aussi d'une suite de syntagmes comme en (15), groupes nominaux en général, parfois ordonnés ou expansés, mais cela reste rare.

Le dernier mot, en aval, peut s'inscrire différemment dans cette suite, à l'enfilade ou en détachement, mais toujours selon deux modes distincts :

Il peut être enchainé aux mots précédents dans un rapport hiérarchique. On relève par exemple dans le corpus des rapports d'analycité comme en (17), d'inclusion en (18) ou d'exemplification en (19) :

(17) On a rendu justice à votre mérite ; on vous a trouvé l'air noble, la démarche aisée, l'esprit charmant, les yeux d'une vivacité... en un mot, une figure adorable. (Crébillon Jolyot de, Lettres de la marquise de $M^{* * *}$ au comte de $R^{* * *}, 1732$ )

(18) Les idées et les images des parlementaires, des ministres, des hauts fonctionnaires, des journalistes, de la "classe politique" en un mot, en diffèrent assez profondément sur certains points [...]. (G. Gurvitch, Traité de sociologie, 1968)

(19) Mais, me direz-vous, et les malades, les femmes enceintes (légitimement), les mères d'enfants en bas âge ou de « familles nombreuses » (comme la nôtre) ? Eh bien, celles-là avaient des cartes spéciales (valables aussi chez le laitier, le boulanger ou le boucher), des rations supérieures (des " tickets » de pain, de viande, de lait), en un mot, des privilèges ${ }^{6}$. (Roubaud, La Boucle, 1993)

Il peut être simplement ajouté à la série, sans hiérarchie sémantique, mais sur un même moule syntaxique, ce qui limite le risque d'éparpillement. Sont alors juxtaposés et enchainés de part et d'autre de la locution, des termes de même nature grammaticale, et potentiellement interchangeables. Si ce n'était la présence du marqueur - et sa position finale - rien ne laisserait supposer que l'adjectif rapide dans l'exemple 20 soit plus pertinent que les autres. Hormis leur valeur méliorative, ces termes n'ont pas de point commun. Le but de la nomination multiple n'est pas d'accumuler du " même » pour renforcer l'acte de nomination, mais de « l'approchant » et du « possible » pour l'affiner, voire de l'inédit ; la nomination au final n'est plus conventionnelle, mais librement choisie, et propre à l'énonciateur.

(20) [...] le Greffier, m'attrape par la manche. Il a quelque chose à me dire, n'est-ce pas... il me trouve intéressant, récupérable, en un mot rapide. (Boudard, 19982)

À terme, et dans les deux cas, le dernier mot apparait comme chargé du sens de tous les précédents : ainsi, le sens du mot rapide ne se réduit plus à son acception en langue «agile, 
véloce », mais s'amplifie en discours du sens des mots précédents, " intéressant, récupérable ». Le mot en discours déborde le mot en langue.

Comme toute figure, cette suite de mots en dit plus qu'une simple nomination. Sa mécanique faite de répétition, d'accumulation et de gradation rentabilise le processus de nomination par des effets de style, mais surtout par cet effet de sens, témoignant du lien personnel qui se tisse entre l'énonciateur, les mots qu'il emploie et la langue.

Enfin, si chacun des termes essayés vient charger de son sens le dernier mot choisi, celui-ci rend compte en retour de leur déficience à nommer juste du premier coup. Figure paradoxale comme l'indique si bien S. Bikialo $(2005,55)$ qui représente « [...] le manque par l'excès, la difficulté de nommer par la prolifération de nominations $»$.

\subsection{Les effets du métaterme : en un mot vs bref et en somme}

La glose de nomination résomptive peut se construire avec d'autres marqueurs. Si en un mot apparait comme le marqueur privilégié de cette classe de gloses, d'autres adverbiaux comme bref et en somme peuvent également tenir ce rôle à l'exemple des extraits 22 et 23.

(21) L'idée que j'aurais pu savoir d'elle-même quelque chose qu'elle n'aurait pas su que je savais, m'inspirait la sombre répulsion qu'engendrent les mauvaises actions, les malhonnêtetés, les tristesses, en somme le vol. (L. Guilloux, Le Pain des rêves, 1942)

(22) Ma situation me dicte de mettre du plus dans la vertu et du moins dans le vice, et d'appeler vertu le courage, la force, l'affirmation de moi-même, la domination sur les choses. Et vice le renoncement, l'abandon, la résignation, bref la souille. M. Tournier, Vendredi ou les Limbes du Pacifique, 1967)

(23) C'est un talent du plus grand caractère. Les descriptions, les narrations, les expressions, le style en un mot, tout est du plus beau genre. (C-J. Chênedollé, Journal de Chênedollé, 1833)

Rien ne semble différencier, dans le format et dans la portée, les deux premières gloses de la troisième. Dans l'architecture de la figure, chacun des marqueurs isole par détachement syntaxique un mot d'une suite de mots et le met au premier plan. Les adverbiaux sont apparemment substituables. En un mot et en somme commutent facilement en position médiane ou finale, et il en est de même pour en un mot et bref en position médiane. Par conséquent, en quoi une glose de nomination résomptive à métaterme se distingue-t-elle d'une glose à terme ordinaire ? que peut bien impliquer dans la glose la présence du marqueur en un mot par rapport à celle des adverbiaux en somme ou bref? Plusieurs paramètres énonciatifs semblent les distinguer :

Tout d'abord la densité de la modalisation autonymique : le marquage de la modalisation autonymique n'a pas la même densité dans l'une et l'autre glose.

Dans les trois extraits, l'énonciation s'effectue en s'auto-représentant en train de se faire. Mais la modalisation autonymique de l'énoncé est explicite et sans équivoque en 23, attestée par le marqueur à métaterme ; elle est contextuelle en 21 et 22, interprétée à l'appui du format syntaxique et figural de l'énoncé. Certes, la relation de couplage qui opère une nomination résomptive est métalinguistique dans les deux cas, mais le dernier mot n'a pas le même statut sémiotique selon le marqueur choisi : mot autonyme avec en un mot; mot ordinaire avec bref ou en somme. Le couplage n'est pas opacifiant ${ }^{7}$ dans ce second cas, sauf à ajouter une marque typographique en renfort. Il est opacifié dans le premier du fait de la catégorisation opérée par le métaterme mettant l'accent sur la matérialité linguistique du terme : « rapide est un mot». 
Ensuite, la potentialité d'une réactivation sémantique du marqueur en un mot : la dimension sylleptique ${ }^{8}$ du marqueur ouvre simultanément la possibilité d'une double interprétation de la séquence : non-compositionnelle, elle sera prise dans sa globalité, celle d'une locution adverbiale, en un mot, opérant une mise en relation à valeur récapitulative au même titre que bref par exemple ; compositionnelle, elle sera saisie dans la combinatoire de ses trois unités, procédant à une quantification et une catégorisation métalinguistique du dernier mot de la liste. Ce double sens, cette syllepse (Chevalier et Wahl, 2006 ; Fontanier [1830] 1977) repose sur la polysémie du terme mot et l'homonymie du déterminant indéfini $u n$ et du déterminant numéral « $1 »$.

(24) Les récits des quatre évangélistes ne sont plus qu'une suite d'allégories, de fables telles que celles d'Ésope et de La Fontaine, des contes et des chants populaires ; en un mot, un mythe. (Quinet, Allemagne et Italie, 1836)

Ainsi, dans cet extrait, la locution fonctionne comme un tout qui connecte logiquement et indexe hiérarchiquement la série " allégories », " fables ", " contes », " chants populaires » au « mythe », mais à tout moment et au moindre indice- ici l'italique qui ne porte que sur le nom - réapparait un sens compositionnel et descriptif où en un mot vaut pour en 1 terme. C'est le mot représenté, l'autonyme affiché graphiquement, mythe, qui rétroactivement réactive le double sens latent du marqueur de glose.

Enfin, ce faisceau de relations, qui augmente le fil du discours d'une épaisseur sémantique, n'est pas toujours perçu d'emblée par lecteur. Ce sont les traces et les reliefs graphiques laissés pour lui par le scripteur qui peuvent l'alerter. Dans le cas des gloses de nomination formées avec bref ou en somme, les mises en relief graphiques autonymisent le dernier mot ; dans celui des gloses faites avec en un mot, ce soulignement s'ajoute à l'autonyme et procède d'un jeu sur le mot.

La signalisation graphique vient en effet en surenchère dans ce cas. Parmi ces signaux, allant des plus transparents aux plus évidents, se trouvent le détachement du mot à droite ou à gauche de la locution qui le met ainsi en exergue et qui est propre à l'architecture de la glose, mais aussi le balisage de la ponctuation - les tirets, les deux points ou les guillemets - qui le délimite ou encore le marquage graphique plus courant - italique ou soulignement qui le matérialise :

(25) Plus modeste, elle est l'apanage des petits étangs assez régulièrement fréquentés par les cols verts, les sarcelles, les foulques, par la "sauvagine" en un mot et dont la présence très temporaire n'incite pas le chasseur à les ménager pour des plaisirs ultérieurs. (Vidron, La Chasse en plaine et au bois, 1945)

(26) Partout on trouve des gens dont le regard vif, étrange, clignotant et faux, le « mauvais œil » en un mot, fait qu'ils sont craints et mal vus. Ils sont tout désignés pour être magiciens. (Mauss, Sociologie et anthropologie, 1950

(27) D'autre part une action extérieure, par exemple la persécution, la faute, la pénitence, le malheur, le doute, en un mot le torcular, exerçant sur nous une action comprimante et resserrante, nous obligent, comme on dit, à rentrer en nous-mêmes. (Claudel, Commentaires et exégèses, 1948)

Cette signalisation du mot se rencontre peu, ou du moins diversement, dans les gloses utilisant les marqueurs bref ou en somme. Le plus souvent, le mot se trouve détaché à l'appui du marqueur, d'une virgule ou des deux points, mais il figure rarement entre guillemets ou en italique. Vraisemblablement, le marquage $d u$ mot n'est pas pertinent dans 
ce cas alors qu'il l'est pleinement dans les exemples ci-dessus. En 26 et en 28, entrant en résonnance avec le marqueur en un mot, les guillemets et l'italique extraient le mot, et non le syntagme, de la linéarité du texte ; en 27 , les guillemets, parant le risque d'un faux sens, délimitent explicitement l'unité lexicale, le mauvais æeil.

Tous ces signaux d'opacification du mot, qui ne peuvent échapper totalement à l'œil du lecteur, aiguillent l'interprétation du lecteur et rapprochent l'énonciateur de son destinataire dans une même connivence face à la langue et à l'infini des discours.

\section{Conclusion}

En conclusion, on soulignera que la locution en un mot est une expression singulière. Elle se caractérise par sa densité métalinguistique et sa polyvalence au sein du discours. Elle peut en effet tenir le rôle d'un modalisateur, d'un connecteur de reformulation non paraphrastique ou d'un marqueur de glose.

Comme marqueur de glose, elle participe d'une glose de nomination complexe à valeur récapitulative. Multiple et progressive, elle se distingue par sa dimension figurale qui charge le mot en discours de plus de sens qu'il n'en a en langue.

Sur le plan énonciatif, cette glose de nomination multiple ne manifeste pas tout à fait les mêmes propriétés énonciatives, selon qu'elle s'effectue avec le marqueur à métaterme en un mot ou les marqueurs simples, bref et en somme. Rappelons, sous forme de tableau, les traits qui les différencient.

\begin{tabular}{|c|c|c|}
\hline $\begin{array}{c}\text { Glose de } \\
\text { nomination résomptive }\end{array}$ & $\begin{array}{l}\text { Marqueur à métaterme } \\
x^{n} \text { en un mot } z\end{array}$ & $\begin{array}{l}\text { Marqueur sans métaterme } \\
x^{n} \text { en somme/bref } z\end{array}$ \\
\hline $\begin{array}{c}\text { Modalisation } \\
\text { autonymique de la glose }\end{array}$ & explicite et sans équivoque & implicite et interprétative \\
\hline $\begin{array}{l}\text { Relation de couplage } \\
\text { entre } x(n) \text { et } z\end{array}$ & $\begin{array}{l}\text { Métalinguistique et } \\
\text { opacifiante } \\
\text { z a de fait un statut } \\
\text { autonyme : } \mathrm{z} \\
>\quad \mathrm{x}^{\mathrm{n}} \text { en un mot } \mathbf{z}\end{array}$ & $\begin{array}{l}\text { Métalinguistique sans } \\
\quad \text { opacification } \\
\mathrm{z} \text { a de fait un statut } \\
\quad \text { ordinaire : } \mathrm{z} \\
>\quad \mathrm{x}^{\mathrm{n}} \text { en somme/bref } \mathbf{z}\end{array}$ \\
\hline $\begin{array}{l}\text { Potentialité du marqueur } \\
\text { de glose }\end{array}$ & $\begin{array}{l}\text { Réactivation sémantique } \\
\text { du marqueur toujours } \\
\text { possible : } \\
\text { en un mot / en } 1 \text { mot } \\
\text { + marquage graphique de } \\
\text { l'autonyme } z \text { ' en } \\
\text { surenchère }\end{array}$ & $\begin{array}{l}\text { Pas de réactivation sémantique du } \\
\text { marqueur } \\
\text { + marquage graphique } \\
\text { d'autonymisation de z possible } \\
\text { mais rare }\end{array}$ \\
\hline
\end{tabular}

Il reste d'autres paramètres que nous n'avons pas pu envisager dans les limites de cette contribution, notamment la distribution de ces trois marqueurs à l'oral et/ou l'écrit. À l'inverse de bref, il semble que la locution en un mot se rencontre prioritairement à l'écrit. Est-ce une spécificité écrite ou un phénomène de registre de langue ? une question de densité métalinguistique plus propice à l'expression de la matérialité graphique ou le signal d'une obsolescence de la locution à l'oral, compensée par les emplois fournis de bref et en somme... L'analyse de ce type de glose et du marqueur en un mot n'est pas épuisée. D'une grande richesse, elle appelle d'autres explorations. 


\section{Références bibliographiques}

Authier-Revuz, J. (1995). Ces mots qui ne vont pas de soi. Boucles réflexives et noncoïncidences du dire. Paris : Larousse.

Authier-Revuz, J. (1994). " L'énonciateur glosateur de ses mots : explicitation et interprétation » Langue française, 103, p.91-102.

Anscombre, J.-C. Donaire M.-L., Haillet, P.-P. (éds) (2013). Opérateurs discursifs du français. Élements de description sémantique et pragmatique. Bern : Peter Lang.

Bikialo, S. (2005). «Plusieurs mots pour une chose. De la nomination multiple au style de Claude Simon ». L'information grammaticale, 104, p.50-53.

Bonhomme, M. (1998). Les figures clés du discours. Berne : Seuil.

Chevalier, Y, Wahl, P. (Dirs) (2006). La syllepse, figure stylistique. Lyon : Presses Universitaires de Lyon.

Dostie, G., Lefeuvre, F. (dirs), (2017). Lexique, grammaire, discours. Les marqueurs discursifs, Paris : Honoré Champion.

Fontanier, P., (1977). Les figures du discours. Paris : Flammarion.

Gómez-Jordana Ferary, S., Anscombre, J.-C. (éds) (2015). Dire et ses marqueurs. Langue française, 186.

Gomila, C. (2019). «En un mot : quel type d'expression métadiscursive ? ». Stu ii e lin istic , 9.2, p.21-39.

Grize, H. P. (1979). « Logique et conversation ». Communication, 30, p. 57-72.

Julia, C. (2001). Fixer le sens ? La sémantique spontanée des gloses de spécification du sens. Paris : Presses Sorbonne Nouvelle.

Lecolle, M. (dir.) (2014). Métalangage et expression du sentiment linguistique "profane". Le discours et la langue, 6, p. 6-18.

Perrin, L. (2012). Modalisateurs, connecteurs et autres formules énonciatives. Lucie Gournay, Lionel Dufaye (éds), Les théories de l'énonciation : Benveniste après un demi-siècle. Paris : Arts et Savoirs 2.

Rey-Debove, J. (1997). Le métalangage naturel : étude linguistique du discours sur le langage. Paris : Armand Colin.

Rossari, C. (1990). « Projet pour une typologie des opérations de reformulation ». Cahiers de linguistique française, 11 , p. 345-359.

Rouanne, L, Anscombre J.-C. (dirs) (2016). Histoire de dire. Petit glossaire des marqueurs formés sur le verbe dire. Bern : Peter Lang. 
Roulet, E. (1987). Complétude interactive et connecteurs reformulatifs. Cahiers de linguistique française. Université de Genève, 8, 111-139.

Steuckardt, A., Niklas-Salminen, A. (dirs) (2003). Le mot et sa glose. Aix-en-Provence : Publications de l'Université de Provence.

Steuckardt, A., Niklas-Salminen, A. (dirs) (2005). Les marqueurs de glose. Aix-enProvence : Publications de l'Université de Provence.

Steuckardt, A., (2006). Du discours au lexique : la glose. Séminaire ATILF, Nancy, France.

${ }^{1}$ Ce travail doit énormément aux travaux de J. Authier-Revuz qui étayent toute notre réflexion.

${ }^{2}$ Le soulignement est de notre fait.

${ }^{3}$ La locution est plus fréquemment employée comme un modalisateur simple au sein d'un énoncé ou un connecteur discursif articulant deux propositions que comme un marqueur de glose.

${ }^{4}$ Nous parlerons plus volontiers de suite, de série ou de succession d'unités que de liste. La liste étant une « suite continue, hiérarchisée ou non, de noms (de personnes ou d'objets) ou de signes généralement présentés en colonne » (TLFI). Ce n'est pas le cas ici.

${ }^{5}$ Comme dans l'extrait suivant :

«Le Docteur, s'obstinant. -moi qui voulais justement vous parler d'un mien neveu... orphelin, dont je me suis beaucoup occupé... un garçon remarquablement doué... jeune, sérieux, très travailleur... en un mot : une perle... » (R. Martin du Gard, Un taciturne, 1932). ${ }^{6}$ Le soulignement n'est pas de notre fait.

${ }^{7}$ Sur la notion d'opacification propre à la modalisation autonymique, voir J. Authier-Revuz (1995, 25-34).

${ }^{8}$ La syllepse consiste, selon Fontanier ([1830] 1977 : 105), «pren re un m me $m$ t $t$ ut-la-fois dans deux sens i rens». 